\section{Reduction of Heart Flavoprotein}

WE recently described ${ }^{1}$ the catalytic properties of a flavoprotein isolated from heart. At that time we were unable to observe the reduction of flavoprotein by reduced coenzyme I. We now find that, by eliminating coloured impurities in the various components of the catalytic system, the rapid reduction of flavoprotein to its leuco form can be readily demonstrated. Under the conditions of our experiment, the leuco form is non-autoxidizable but can be oxidized by 'carriers' such as methylene blue. The mechanism of the catalytic effect of heart flavoprotein involves a cycle of reduction by reduced coenzyme I and oxidation of the leuco form by some suitable 'carrier'.

F. B. Straub.

H. S. Corran D. E. Green.

Molteno and Biochemical Institutes, Cambridge.

Feb. 7.

1 Straub, Corran and Green, NATURe, 143, 119 (1939).

\section{Relative Sexuality in Unicellular Algæ}

IN a series of papers, Dr. Franz Moewus, working first at the Kaiser Wilhelm Institut für Biologie in Berlin, and later at Erlangen and Heidelberg, has published a number of very remarkable results on relative sexuality in unicellular algæ. The qualitative results are in agreement with those of other workers of the same school. We wish, however, to direct attention to a feature of his quantitative data which casts grave doubt on their reliability.

Moewus' last paper ${ }^{1}$ described the genetical behaviour of various races of Chlamydomonas eugametos. The zygotes derived from crosses within diœcious races, that is, races with genotypical sex-determination, are said to give 3.08 per cent exceptional gametes. Clones grown from these exceptional gametes can produce gametes of both sexes, that is, they show phenotypical sex-determination. These abnormal segregations are ascribed to crossing over between the two non-allelomorphic sex determiners. In each of the crosses 1-9, 18-34, and 37-40 he tested 500 , or occasionally 400 or 300 zygotes. In 27 of the 31 crosses, 500 zygotes were tested, and the number of exceptions was between 13 and 17 in every case.

Struck by this astonishing consistency, we applied Pearson's $\chi^{2}$ test to all 31 crosses, and found $\chi^{2}=$ 2.423 for thirty degrees of freedom. The probability of obtaining as bad or worse a fit by sampling is $P=1-4.7 \times 10^{-12}$. That is to say, only once in about $10^{11}$ trials would so good a fit be obtained by chance. In the same paper (Table 7) Moewus once more calculates the above "cross-over" value from four samples of 5,000 zygotes each, by observing the number of cells dying as a result of meiosis. The numbers are 152, 158, 151, and 155, forming exactly 3.08 per cent of the total, as before. We can therefore add the total value of $\chi^{2}$ obtained from them, namely, $0 \cdot 201$ for four more degrees of freedom, to the former value, obtaining a grand total of $\chi^{2}=$ $2 \cdot 624$ for thirty-four degrees. This gives $P=1-$ $6.6 \times 10^{-14}$.

A second suggested cross-over frequency between the male determiner $M$ and the gene $t$ for Subhetercecie amounting to $9 \cdot 8$ per cent is deduced from six crosses (numbers 36-39 and 42-43). Here $\chi^{2}=0 \cdot 0410$ for five degrees of freedom. So $P=1-6 \cdot 3 \times 10^{-6}$ approximately, though even Sheppard's table of the probability integral is not accurate enough to give the second significant figure with certainty.

Nor is the truly remarkable agreement between theory and observation confined to the paper in question. In a paper ${ }^{2}$ on Protosiphon botryoides, Tables 2, 3, and 4 record twenty-two experiments in which the cultures segregated into approximately equal numbers. Either the two halves gave different kinds of gamete or one gave gametes and the other did not. In eleven experiments where thirty cultures were counted, the deviation from equality never exceeded 17 and 13 . In eleven experiments where ten cultures were counted, the largest deviation is 6 and 4. For twenty-two degrees of freedom $\chi^{2}=$ $5 \cdot 06, P=1-6.9 \times 10^{-5}$.

So good a general agreement with theory would be obtained by chance once in $3.5 \times 10^{22}$ trials; that is to say, if every member of the human race conducted a set of experiments of this type daily, they might reasonably hope for such a success once in fifty thousand million years. There are two alternatives to the hypothesis of chance. One is that a wholly new type of biological regulation has been unwittingly discovered, by which the frequency of segregation is controlled with extreme sharpness, and that this applies to three segregations in two species. The other is that the author has consciously or unconsciously adjusted his observations to fit his theory. As this work is of fundamental importance for the theory of sex-determination, it is desirable that these experiments should be repeated by an independent observer. Until this is done, those parts of Dr. Moewus' genetical theory which are based on these numbers cannot, we think, be accepted.

\section{Department of Biometry, University College,} U. PHILIP. London. Jan. 25.

Biol. Zbl., 58, 516-536 (1938).

¿ Biol. Zbl., 55, 293-309 (1935).

\section{Embryology of the Symphyla}

OWING to the significance which is attached to the Symphyla in speculations on the origin of insects, a knowledge of their embryology has become desirable. Having recently obtained, in large quantity, the eggs of a local species of Hanseniella, I have been able to work out a fairly complete story of the development of these remarkable animals. A detailed account of this will be published later; the present note records a few points which are significant on phylogenetic grounds.

Cleavage is total, as in Collembola and some Diplopoda. By tangential division of the large yolkpyramids, an outer layer of small cells and an inner layer of very large cells arises, both rich in yolk. From the former arises the blastoderm. From the latter arises by further division the internal mass of yolk-cells. These are not pure vitellophages, however, for from them develop the fat-body and mid-gut epithelium.

An embryonic area forms by ventral thickening of the blastoderm. A precocious flexure then forms in the embryonic area, as in some diplopods ${ }^{1}$, and thereby the germ-band becomes defined. A 'dorsal organ' is present; but there are no embryonic membranes. 